\title{
Wolbachia and arbovirus inhibition in mosquitoes
}

\section{Steven P Sinkins}

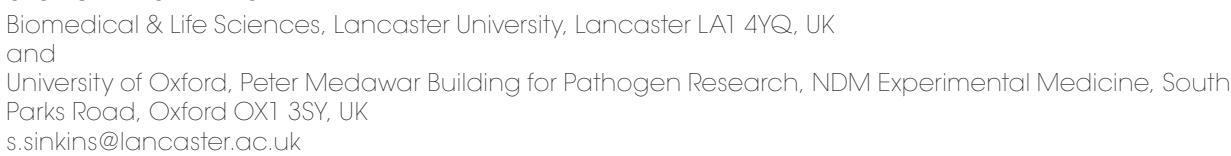

Wolbachia is a maternally inherited intracellular bacteria that can manipulate the reproduction of their insect hosts, and cytoplasmic incompatibility allows them to spread through mosquito populations. When particular strains of Wolbachia are transferred into certain Aedes mosquito species, the transmission capacity of important arthropod-borne viruses can be suppressed or abolished in laboratory challenges. Viral inhibition is associated with higher densities of transinfecting Wolbachia compared with wild-type strains of the bacterium. The upregulation of innate immune effectors can contribute to virus inhibition in Aedes aegypti, but does not seem to be required. Modulation of autophagy and lipid metabolism, and intracellular competition between viruses and bacteria for lipids, provide promising hypotheses for the mechanism of inhibition. Transinfecting virus-inhibiting strains can produce higher fitness costs than wild-type mosquito Wolbachia; however, this is not always the case, and the wMel strain has already been introduced to high frequency in wild Ae. aegypti populations.

\section{Arbovirus inhibition phenotype}

Arboviruses transmitted by mosquitoes have great importance in global health, owing to increasing ranges and impact and often with no effective vaccines or reliable prophylactics available. The most important is the flavivirus dengue virus (DENV), which occurs in 100 countries and causes tens of millions of cases of dengue fever annually, approximately half a million of which proceed to life-threatening hemorrhagic fever or shock syndrome [1]. Chikungunya virus (CHIKV), an alphavirus, is an important emerging pathogen in many regions of the world, and a number of epidemics with tens of thousands of cases or more have occurred in the last decade [2]. A dramatic transmission-blocking phenotype (e.g., no infectious viral particles could be detected in the mosquito saliva) for both viruses was observed in the mosquito Aedes aegypti, the most important vector of DENV. This vector was artificially transinfected with the $w \mathrm{MelPop}$ and $w \mathrm{Mel}$ strains of the bacterial endosymbiont Wolbachia from Drosophila melanogaster [3-5]; inhibition of yellow fever virus was also observed in $w \mathrm{Mel}$-infected Ae. aegypti [5]. A $w \mathrm{Mel}$ transinfection in Aedes albopictus blocked the laboratory transmission capacity for DENV [6] and CHIKV [7]. This species is often considered a secondary vector of DENV, although it has been the primary or sole vector in a number of epidemics, and is frequently a primary vector of CHIKV following an envelope protein mutation that significantly and specifically increased viral fitness in Ae. albopictus [8,9].
Interestingly, Wolbachia can also inhibit the transmission of some mosquito-borne parasites, such as Plasmodium by Ae. aegypti [3].

Wolbachia are intracellular and tend to be most concentrated in reproductive organs, but often have a wide tissue distribution within the host insect, including midgut and salivary glands. Arboviruses acquired in the blood meal must invade midgut cells and ultimately disseminate to the salivary glands to be transmitted, so Wolbachia and the virus can potentially be present within the same cells. Wolbachia itself is not an infectious agent, but is maternally transmitted in the egg cytoplasm and is able to invade host populations by manipulating their reproduction. The most widely observed reproductive phenotype (and the only one observed in mosquitoes) involves crossing sterilities known as cytoplasmic incompatibility. In the simplest case, consider crosses between Wolbachia-infected and uninfected individuals, early embryonic arrest occurs when uninfected females mate with infected males [10]; however, the offspring of infected females develop normally regardless of the male infection status, thus giving infected females a reproductive advantage. The self-spreading ability of Wolbachia makes it an attractive biocontrol agent to interrupt or reduce arbovirus transmission. A first field trial targeting wild populations of Ae. aegypti in northern Australia has proven successful in introducing the $w$ Mel strain to high population frequency [11].

A better understanding of the mechanisms of inhibition of DENV and CHIKV by Wolbachia
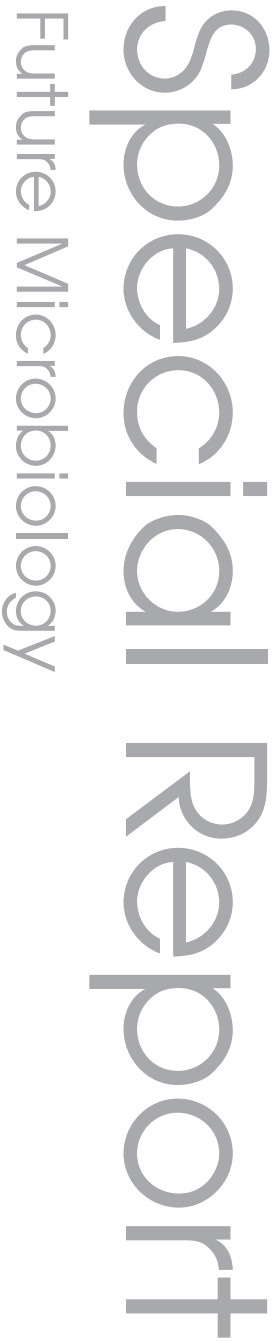

\section{Keywords}

- Aedes = autophagy - chikungunya = dengue - endosymbiont a innate immunity = insect - lipids - West Nile virus

\section{Future} Medicine part of 
is important in order to make informed predictions of the selective forces that will operate on the virus and mosquito that might ameliorate the inhibition phenotype over time, and thus how to maximize the effectiveness and longevity of the strategy. What is known so far and what hypotheses can be generated regarding how the viral inhibition phenotype works (FIgure 1) will be considered here, together with the effects of different Wolbachia strains on host fitness, which is an important factor with respect to Wolbachia population invasion dynamics.

\section{Strain specificity of viral inhibition \& Wolbachia density}

The viral inhibition phenotype is Wolbachia strain-dependent, and there is also an effect of host species on the degree of inhibition afforded. Wolbachia is naturally present in many mosquito species. Wild Ae. albopictus individuals usually carry two strains of the bacterium [12], wAlbA and $w \mathrm{AlbB}$, and a modulating effect on transmission capacity has been reported for DENV compared with antibiotic-treated Wolbachia-free mosquitoes of this species [13]; although, this difference was not apparent in all experiments and was much more modest than that observed when wMel strain Wolbachia was introduced [6]. Interestingly, high experimental CHIKV titers produced a reduction in density of wild-type wAlbA and wAlbB Wolbachia in Ae. albopictus [14], which suggests resource competition between bacteria and virus. When the wAlbB strain was transferred from Ae. albopictus into Ae. aegypti, stronger inhibition of DENV midgut replication and subsequent dissemination to thorax and head was observed compared with in the natural host [15]; however, the degree of inhibition was still lower than that produced by the $w$ MelPop transinfection in Ae. aegypti [3]. In addition wild-type Culex quinquefasciatus, carrying the $w$ Pip-strain of Wolbachia, showed reduced dissemination of West Nile virus (WNV) when compared with an uninfected, antibiotic-cured line. WNV is a zoonotic flavivirus causing increasing numbers of cases of human encephalitis, which is endemic in Africa, Europe, central Asia and Australia,

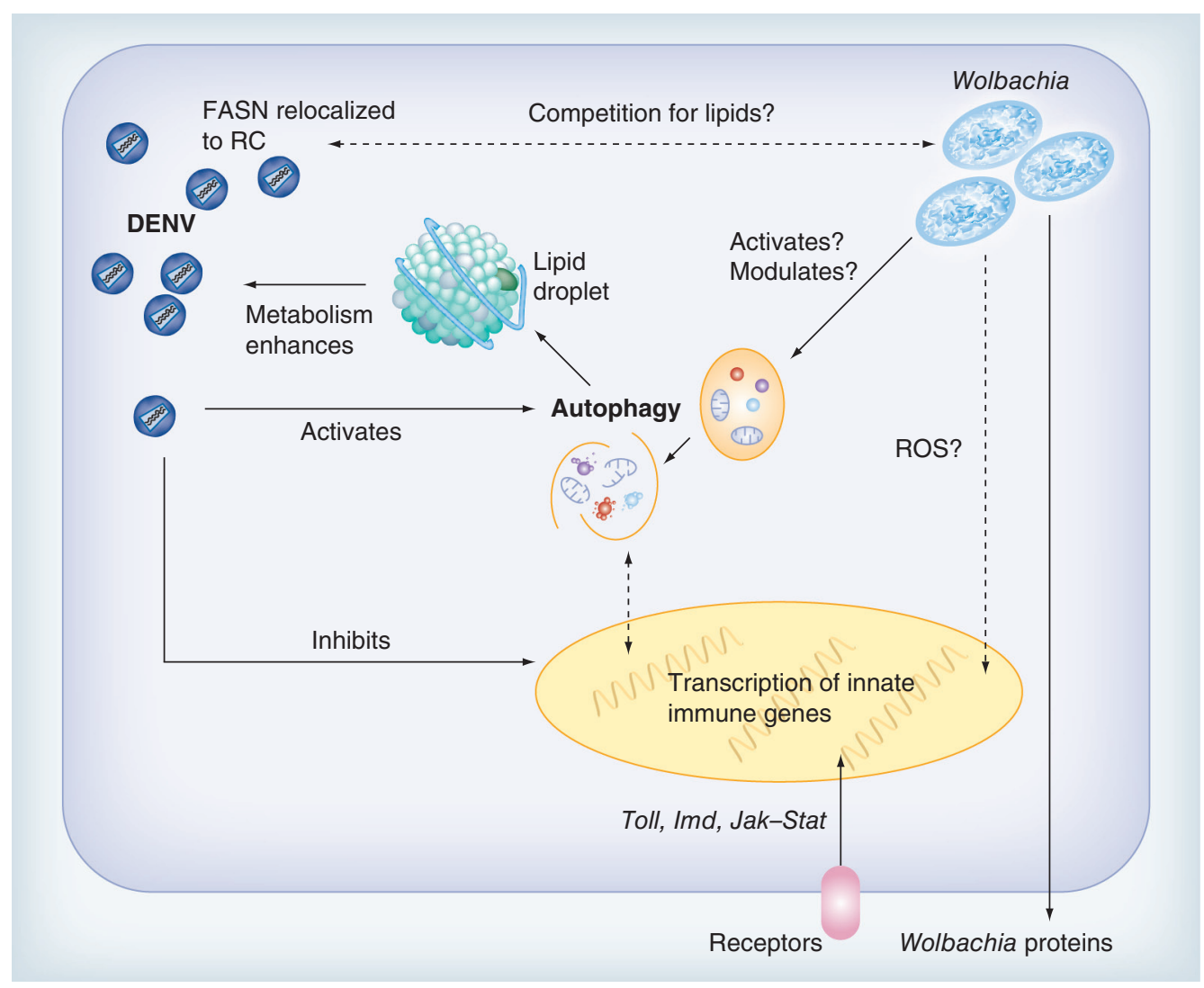

Figure 1. Some of the interactions between high-density Wolbachia infections and dengue virus in mosquito cells that could contribute to the arbovirus inhibition phenotype.

Interactions that are more uncertain in terms of the effects or direction are shown as hatched lines. Oxidative stress was induced by the presence of high densities of Wolbachia.

DENV: Dengue virus; FASN: Fatty acid synthase; RC: Viral replication complex; ROS: Reactive oxygen species. 
and has recently expanded its range into North America [16].

A number of studies have now shown that the transinfecting Drosophila strains of Wolbachia reach higher densities in mosquitoes than the naturally occurring mosquito Wolbachia infections, and there is evidence that high density is causally linked to the virus inhibition phenotype. A direct comparison of $w \mathrm{Mel}$ and wild-type $w \mathrm{AlbA} / w \mathrm{AlbB}$ in Ae. albopictus, in a genetically homogenous background, showed approximately a tenfold higher overall density reached by $w \mathrm{Mel}$ [6]. An association of degree of inhibition of viral replication with bacterial density or load was observed in cell lines for both DENV and WNV [17-19]. In wMelPopinfected Ae. aegypti challenged with DENV, a nonoverlapping distribution of bacterial and viral particles was reported within tissues, suggesting a cell-autonomous antiviral effect [3]. In parallel studies in D. melanogaster and Drosophila simulans, some but not all Wolbachia strains provided protection against pathogenic viruses such as DCV (associated with a reduction in viral titers) and also flock house virus and Nora virus (not associated with a decrease in viral titers); only the higher density Wolbachia strains afforded virus protection [20-23]. Indeed, when the density of one of the strains showing high viral protection was reduced using antibiotics to levels similar to that of the nonprotective strains, the viral protection phenotype was lost [23]: this provides direct evidence of the importance of bacterial density to the phenotype.

\section{Mosquito innate immune pathways}

The transinfection of $w$ MelPop Wolbachia in Ae. aegypti produced a chronic, major upregulation of a number of immune genes, including components of the Toll, Imd, Jak-Stat and melanization pathways and their effectors, such as cecropins and defensins, and thioester-containing proteins $[3,24]$. Lower level upregulation of selected immune genes was observed for the $w$ AlbB transinfection in Ae. aegypti [16]. The Toll pathway in particular is known to be activated in mosquitoes by DENV and to modulate the replication of the virus. In addition, the presence of the gut microbiota influences viral infection levels via basal Toll pathway immune activation [25]. The activation of the Ae. aegypti Toll pathway in the line transinfected with $w \mathrm{AlbB}$, has been reported to be mediated through the production of reactive oxygen species and activation of the NADPH oxidase, resulting in upregulation of Toll pathway cecropins and defensins. It has been demonstrated using knockdowns and transgenic lines that levels of these antimicrobial peptides can influence DENV titers [26]. Thus, the presence of Wolbachia in Ae. aegyptican allow the primed mosquito immune system to eliminate virus particles more effectively. It also seems likely that the enhanced immune response allows more efficient killing of parasites, such as filarial nematodes and Plasmodium [24,27].

However, in an Ae. albopictus line transinfected with $w \mathrm{Mel}$, a selection of orthologs of immune effector genes that were upregulated in the presence of Wolbachia in Ae. aegypti, including a cecropin and defensin, were not significantly upregulated at generation ten [6]. Given that this mosquito naturally carries Wolbachia, it would seem that a long evolutionary history of association has modulated its immune response, even to a non-native strain such as $w \mathrm{Mel}$. Whether or not the host naturally harbors Wolbachia appears to be the important factor in the different immune pathway responses, rather than the differing densities reached by natural versus transinfecting strains. While the involvement of immune genes in virus inhibition has not been ruled out, since an exhaustive screen has not yet been carried out for this Ae. albopictus transinfection, it does appear that other factors beyond the mosquito innate immune system are primarily responsible for the blockage of DENV and CHIKV transmission (whereby no infectious viruses were detected in the saliva) in Ae. albopictus; Wolbachia strain type and density reached are the important factors here. In parallel, the low or insignificant induction of AMPs and other Toll and Imd pathway immune genes observed in Wolbachia-infected Drosophila [28,29] has suggested that other mechanisms beyond the canonical immune pathways must be operating to provide viral protection.

The mosquito antiviral RNA interference response has been shown to be a significant determinant of the efficiency of DENV transmission by Ae. aegypti [30]. However, the presence of Wolbachia reduces DENV replication in the Ae. albopictus C6/36 cell line [17], and this cell line shows an impaired RNA interference antiviral response, lacking functional Dicer-2 expression [31], suggesting that this pathway of viral defense is unlikely to be an important component of the phenotype [32].

\section{Autophagy, lipid metabolism \& cholesterol}

Autophagy is the essential and ubiquitous lysosomal degradation pathway by which eukaryotic 
cells dispose of organelles and large protein aggregates, especially in times of stress and starvation, and for recycling cellular components during development. It is also used to degrade bacteria, viruses and protozoan parasites that invade cells, and the autophagy machinery thus interfaces with innate immune response pathways [32]. Despite its antiviral functions as a branch of the immune system, autophagy can also be subverted by some viruses to perform proviral roles. DENV has been shown to both induce and require autophagy for robust viral replication in mammalian cells [33,34], specifically of a form of autophagy that is targeted to lipid droplets, producing changes in the metabolism of the cell. The DENV-induced autophagosomes colocalize with lipid droplets and become autolysosomes, leading to a release of free fatty acids. DENV replication inhibition, caused by inhibition of autophagy, is rescued by the addition of free fatty acids with $\beta$-oxidation; thus, this pathway is believed to provide a favorable environment for viral replication by generating energy $[35,36]$. CHIKV has likewise been shown to activate autophagy, and this process promotes its replication [37]. Lipid droplets have also been proposed to be involved in DENV particle assembly [38].

The intracellular bacterium Anaplasma phagocytophilum, which is related to Wolbachia, secretes a protein that hijacks the autophagy initiation pathway, probably as a mechanism to acquire host nutrients [39]. It has recently been demonstrated that a protein that provides a marker of activation of autophagy, ATG8a, is abundant in Brugia tissues in which Wolbachia is abundant. In addition, autophagy activation using rapamycin can reduce Wolbachia density in Ae. albopictus cells [40]. Experiments are now needed to determine the ways in which Wolbachia may modulate autophagy to establish its intracellular niche, and whether that modulation could influence arboviral interactions with the autophagy pathways. Autophagy is a complex process and it is possible that the autophagyrelated interactions of these viruses observed so far in mammalian cells will prove to be specific to the mammalian host. Experiments investigating how DENV and CHIKV may modulate autophagy in mosquito cells are now needed.

Intracellular membranes are modified by DENV to form the sites of viral replication complexes, and the virus makes significant modifications to the lipid repertoire of infected mosquito cells [41]. DENV has been shown to co-opt the fatty acid biosynthetic pathway, increasing the rate of biosynthesis, for this purpose. Fatty acid synthase, a key enzyme in the pathway, is required by the virus and is localized to the sites of DENV replication in both mammalian [35] and mosquito cells [41]. Wolbachia requires unsaturated fatty acids from host cells, since it lacks fatty acid desaturation genes, and at high bacterial densities fatty acid depletion could represent a cellular perturbation that disturbs the viral manipulation of these pathways. DENV replication is also modulated by cholesterol biosynthesis [42], and alphaviruses such as CHIKV also require cholesterol and sphingolipids for optimal endosomal fusion [43]. Cholesterol is obtained by insects from their diet and is a limiting resource. Wolbachia, like Anaplasma, lack genes for lipopolysaccharide biosynthesis, so may need to incorporate cholesterol derived from host cells into their membranes to ensure their integrity. It has therefore been hypothesized that the presence of Wolbachia at high densities in relevant tissues could inhibit these viruses by depleting cholesterol levels, through direct intracellular competition for this resource [3]. Experimental testing of this hypothesis is needed.

\section{Strain density \& host fitness}

The dynamics of Wolbachia population replacement using cytoplasmic incompatibility as a disease biocontrol strategy depend in part on any fitness costs of transinfecting Wolbachia strains, which will raise the unstable equilibrium frequency that represents a threshold that must be exceeded for spread to occur. Full characterization of the comparative fitness of each transinfection is of fundamental importance in determining which strains should be used for biocontrol, and to optimize efficiency. The viral inhibition phenotype itself is unlikely to provide any major selective advantages with respect to protection from arboviruses, such as DENV, which do not impose major fitness costs on the mosquito [44]. The extent of any fitness advantages that could be accrued through protection from other mosquito pathogens is largely unknown.

In Ae. aegypti, the high density $w$ MelPop strain is virulent and approximately halves the lifespan of the Ae. aegypti host [45,46]. This life-shortening is advantageous with respect to disease control, since a viral extrinsic incubation period is required within the mosquito before it becomes infectious, such that reducing the proportion of older females will have a disproportionate effect in reducing virus transmission [45]. In theory $w$ MelPop could still spread through populations if fitness costs are primarily incurred by older individuals, 
since most reproduction is undertaken by young females in nature, only a minority would live long enough for the additional fitness load imposed by $w$ MelPop to have a significant negative effect. Indeed, invasion of cage populations of Ae. aegypti by $w$ MelPop has been documented [4]. However, the $w$ MelPop transinfection also decreased the viability of stored Ae. aegypti eggs over time [5,47]. Desiccated eggs often play an important role in population maintenance during dry periods, so this could be an important impediment to population spread. Female feeding was also negatively affected in some individuals, primarily (but not only) in older $w$ MelPop-infected females, with a 'bendy proboscis' phenotype and difficulty in completing the process of blood feeding effectively (i.e., smaller blood meals taken and lower saliva volumes) [48,49]. Susceptibility to predation (using six different predator types) was not, however, significantly increased in laboratory tests of $w$ MelPop-infected individuals compared with uninfected wild-types [50].

The $w \mathrm{Mel}$ transinfection is considerably more benign in Ae. aegypti in the laboratory [4], without a significant life-shortening phenotype, and its successful introduction into wild populations of $A$ e aegypti confirmed its utility under natural conditions [5]. Male mating competitiveness was not found to be significantly impaired by either the $w \mathrm{Mel}$ or the $w \mathrm{MelPop}$ transinfection in laboratory cage challenges [51]. Assuming the viral inhibition phenotype produced by $w \mathrm{Mel}$ remains as strong in natural populations as in laboratory challenges, then it will clearly be preferable to use this strain for control of disease transmission by Ae. aegypti over wMelPop. In Ae. albopictus the difference was even more pronounced: greatly reduced hatch occurred in eggs laid by females transinfected with $w$ MelPop [52], likely to preclude its use for disease control in this species. However, replacing the wild-type $w \mathrm{AlbA}+w \mathrm{AlbB}$ infection in Ae. albopictus with $w \mathrm{Mel}$ did not produce significant reductions in egg hatch from transinfected females, and no major fitness costs in laboratory cage assays of fecundity, longevity and relative male mating competitiveness [7].

The fitness effects of different Wolbachia strains show a correlation with the overall density reached; however, more specifically, there could be differences in fitness costs even where the overall density reached is similar, according to the range of tissues that are colonized by different strains; for example, the degree of proliferation in nervous tissues. It is possible that there may prove to be trade-offs between the density reached by different strains, degree of viral inhibition achieved under field conditions and fitness costs. It is also important to note that strategies for using Wolbachia in disease control, even strains that cause fitness costs, and thus have higher threshold release frequencies that must be exceeded, can be designed to reduce the population of biting females at the same time. Male-biased releases, where these males are incompatible with wild females [53], can be achieved by pupal size sorting.

\section{Conclusion \& future perspective}

It is likely that a much better understanding of the mechanisms of Wolbachia inhibition in mosquitoes will soon emerge, as experiments to manipulate various genes and cellular pathways are undertaken. There is already evidence that more than one factor can contribute to the phenotype; innate immune effector upregulation can reduce viral replication in Ae. aegypti, but in other systems it is not required for strong virus inhibition to occur. Thus it will be important to not generalize between Drosophila models and

\section{Executive summary}

\section{Arbovirus inhibition}

- Transinfecting Wolbachia can block dengue and chikungunya virus transmission.

- Arbovirus inhibition is associated with higher density compared with wild-type mosquito Wolbachia.

Innate immune upregulation priming

- Can play a role in viral inhibition.

- Not observed in all cases of viral inhibition/protection, thus apparently not required.

\section{Autophagy \& lipid metabolism}

- Modulation of lipid pathways or competition for resources required by both bacteria and viruses could be the cause of viral inhibition.

\section{Fitness effects}

- wMelPop strain can have high associated fitness cost, whereas wMel has much less so.

\section{Conclusion}

- A promising new control strategy for dengue and chikungunya virus.

Wolbachia-based strategies could be applied to control other arboviruses. 
mosquitoes, or between mosquito species, which differ in their immune response to Wolbachia, or between different viruses given the complexity of viral cellular interactions with the host. Cell lines have a useful role to play in these experiments as simplified model systems; in some cases, lacking functional immune pathways. Regulation of lipid metabolism is an important component of DENV and CHIKV replication, and investigations of the effects of Wolbachia on these processes are needed in mosquitoes. Viruses can also modulate or usurp a range of other host cell functions to facilitate their replication (for example, the suppression of apoptosis), and Wolbachia may do likewise. A better understanding of these interactions could provide new hypotheses for the inhibition phenotype. There is also emerging evidence that the presence of Wolbachia changes transcription levels of a host miRNA, and consequently a methyltransferase gene in Ae. aegypti. In addition, levels of expression of methyltransferase could have some influence on DENV replication [54]. Research is needed to determine to what extent this could contribute to arboviral inhibition phenotypes.

The extension of initial open field release trials to other Ae. aegypti populations and into Ae. albopictus will give a much more complete understanding of the utility of this system for disease control, in particular whether complete viral inhibition is always maintained under field conditions and whether the phenotype might be modulated over time. Wolbachia could be used for the control of other arboviruses if transinfections also cause strong inhibition, such as WNV, the encephalitis viruses (Japanese encephalitis, eastern equine encephalitis, Venezuelan equine encephalitis, Saint Louis encephalitis and Murray Valley encephalitis), Rift Valley fever, Ross River virus and Sindbis. All have multiple mosquito vectors and it is unlikely that it will be possible to prevent transmission by them all, given that Wolbachia is solely maternally transmitted and creating transinfections by embryo microinjection and selection is a challenging and labor-intensive process. However, individual important vector species provide attractive potential targets, such as members of the Aedes scutellaris group (regional vectors of DENV and CHIKV), and Culex species such as Culex quinquefasciatus (WNV and others) and Culex triteaniorhynchus (Japanese encephalitis virus). Laboratory cage studies, field cage trials and, most usefully, open field release studies will give the best indication of population dynamics of transinfections and their effectiveness for arbovirus control. Where there are trade-offs between high-density Wolbachia infections needed to produce strong viral inhibition, and fitness costs that may be imposed by the highest Wolbachia loads, the strain-host combinations most useful for arbovirus control will balance these factors.

\section{Financial \& competing interests disclosure}

The author was supported by Wellcome Trust grant 095121. The author has no other relevant affliations or financial involvement with any organization or entity with a financial interest in or financial conflict with the subject matter or materials discussed in the manuscript apart from those disclosed.

No writing assistance was utilized in the production of this manuscript.

\section{Open Access}

This work is licensed under the Creative Commons Attribution-Non Commercial 3.0 Unported License. To view a copy of this license, visit http://creativecommons.org/licenses/by-nc-nd/3.0/

- Reports the creation and characterization of a $w \mathrm{Mel}$ transinfection in Aedes albopictus and shows that it strongly inhibits dengue.

7. Blagrove MSC, Arias-Goeta CA, Cristina Di Genua C, Failloux AB, Sinkins SP. A Wolbachia $w \mathrm{Mel}$ transinfection in Aedes albopictus is not detrimental to host fitness and inhibits chikungunya virus. PLoS Negl. Trop. Dis. 7, e2152 (2013).

8. Tsetsarkin KA, Weaver SC. Sequential adaptive mutations enhance efficient vector switching by chikungunya virus and its epidemic emergence. PLoS Pathog. 7, e1002412 (2011).

6. Blagrove MSC, Arias-Goeta CA, Failloux AB, Sinkins SP. The Wolbachia strain $w \mathrm{Mel}$ induces cytoplasmic incompatibility in and blocks dengue transmission by Aedes albopictus. Proc. Natl Acad. Sci. USA 109, 255-260 (2012).
9. Tsetsarkin KA, Chen R, Sherman MB, Weaver SC. Chikungunya virus: evolution and genetic determinants of emergence. Curr. Opin. Virol. 1, 310-317 (2011). 
10. Serbus LR, Casper-Lindley C, Landmann F, Sullivan W. The genetics and cell biology of Wolbachia-host interactions. Annu. Rev. Genet. 42, 683-707 (2008).

11. Hoffmann AA, Montgomery BL, Popovici J et al. Successful establishment of Wolbachia in Aedes populations to suppress dengue transmission. Nature 476, 454-457 (2011).

- Demonstrates, using an open field trial, that the $w$ Mel strain can be taken to high population frequency in Ae. aegypti, validating its potential as an arbovirus control tool.

12. Sinkins SP, Braig HR, O’Neill SL. Wolbachia superinfections and the expression of cytoplasmic incompatibility. Proc. Biol. Sci. 261, 325-330 (1995).

13. Mousson L, Zouache K, Arias-Goeta C et al. The native Wolbachia symbionts limit transmission of dengue virus in Aedes albopictus. PLoS Negl. Trop. Dis. 6, e1989 (2012).

14. Mousson L, Martin E, Zouache K et al. Wolbachia modulates chikungunya replication in Aedes albopictus. Mol. Ecol. 19, 1953-1964 (2010).

15. Bian G, Xu Y, Lu P, Xie Y, Xi Z. The endosymbiotic bacterium Wolbachia induces resistance to dengue virus in Aedes aegypti. PLoS Pathog. 6, e1000833 (2010).

- Demonstrates that the $w \mathrm{AlbB}$ strain of Wolbachia from Ae. albopictus can reduce dengue virus vector competence when transferred to Ae. aegypti.

16. Glaser RL, Meola MA. The native Wolbachia endosymbionts of Drosophila melanogaster and Culex quinquefasciatus increase host resistance to West Nile virus infection. PLoS ONE 5, e11977 (2010).

17. Frentiu FD, Robinson J, Young PR, McGraw EA, O'Neill SL. Wolbachia-mediated resistance to dengue virus infection and death at the cellular level. PLoS ONE 5, e13398 (2010).

18. Lu P, Bian G, Pan X, Xi Z. Wolbachia induces density-dependent inhibition to dengue virus in mosquito cells. PLoS Negl. Trop. Dis. 6 , e1754 (2012).

19. Hussain M, Lu G, Torres S et al. Effect of Wolbachia on replication of West Nile virus in a mosquito cell line and adult mosquitoes. J. Virol. 87, 851-858 (2013).

20. Teixeira L, Ferreira A, Ashburner M. The bacterial symbiont Wolbachia induces resistance to RNA viral infections in Drosophila melanogaster. PLoS Biol. 6, e1000002 (2008).

21. Hedges LM, Brownlie JC, O’Neill SL, Johnson $\mathrm{KN}$. Wolbachia and virus protection in insects. Science 322, 702 (2008).
22. Osborne SE, Leong YS, O’Neill SL, Johnson $\mathrm{KN}$. Variation in antiviral protection mediated by different Wolbachia strains in Drosophila simulans. PLoS Pathog. 5 , e1000656 (2009).

23. Osborne SE, Iturbe-Ormaetxe I, Brownlie JC, O’Neill SL, Johnson KN. Antiviral protection and the importance of Wolbachia density and tissue tropism in Drosophila simulans. Appl. Environ. Microbiol. 78, 6922-6929 (2012).

24. Kambris Z, Cook PE, Phuc HK, Sinkins SP. Immune activation by life-shortening Wolbachia and reduced filarial competence in mosquitoes. Science 326, 134-136 (2009).

25. Xi Z, Ramirez JL, Dimopoulos G. The Aedes aegypti toll pathway controls dengue virus infection. PLoS Pathog. 4, e1000098 (2008).

26. Pan X, Zhou G, Wu J et al. Wolbachia induces reactive oxygen species (ROS)dependent activation of the toll pathway to control dengue virus in the mosquito Aedes aegypti. Proc. Natl Acad. Sci. USA 109, E23-E31 (2012).

27. Kambris Z, Blagborough AM, Pinto SB et al. Wolbachia stimulates immune gene expression and inhibits Plasmodium development in Anopheles gambiae. PLoS Pathogens 6, e1001143 (2010).

28. Rancès E, Ye YH, Woolfit M, McGraw EA, O'Neill SL. The relative importance of innate immune priming in Wolbachia-mediated dengue interference. PLoS Pathog. 8 , e1002548 (2012).

29. Wong ZS, Hedges LM, Brownlie JC, Johnson KN. Wolbachia-mediated antibacterial protection and immune gene regulation in Drosophila. PLoS ONE 6, e25430 (2011).

30. Sánchez-Vargas I, Scott JC, Poole-Smith BK et al. Dengue virus type 2 infections of Aedes aegypti are modulated by the mosquito's RNA interference pathway. PLoS Pathog. 5 , e1000299 (2009).

31. Brackney DE, Scott JC, Sagawa F et al. C6/36 Aedes albopictus cells have a dysfunctional antiviral RNA interference response. PLoS Negl. Trop. Dis. 4, e856 (2010).

32. Merkling SH, van Rij RP. Beyond RNAi: antiviral defense strategies in Drosophila and mosquito. J. Insect Physiol. 59, 159-170 (2013).

33. Lee YR, Lei HY, Liu MT et al. Autophagic machinery activated by dengue virus enhances virus replication. Virology 374 , 240-248 (2008).

34. Khakpoor A, Panyasrivanit M, Wikan N, Smith DR. A role for autophagolysosomes in dengue virus 3 production in HepG2 cells. J. Gen. Virol. 90, 1093-1103 (2009).
35. Heaton NS, Perera R, Berger KL et al. Dengue virus nonstructural protein 3 redistributes fatty acid synthase to sites of viral replication and increases cellular fatty acid synthesis. Proc. Natl Acad. Sci. USA 107, 17345-17350 (2010).

36. Heaton, NS, Randall G. Dengue virusinduced autophagy regulates lipid metabolism. Cell Host Microbe 8, 422-432 (2010).

37. Krejbich-Trotot P, Gay B, Li-Pat-Yuen G et al. Chikungunya triggers an autophagic process which promotes viral replication. Virol. J. 8, 432 (2011).

38. Samsa MM, Mondotte JA, Iglesias NG et al. Dengue virus capsid protein usurps lipid droplets for viral particle formation. PLoS Pathog. 5, e1000632 (2009).

39. Niu H, Xiong Q, Yamamoto A, HayashiNishino M, Rikihisa Y. Autophagosomes induced by a bacterial Beclin 1 binding protein facilitate obligatory intracellular infection. Proc. Natl Acad. Sci. USA 109, 20800-20807 (2012).

40. Voronin D, Cook DA, Steven A, Taylor MJ. Autophagy regulates Wolbachia populations across diverse symbiotic associations. Proc. Natl Acad. Sci. USA 109, E1638-E1646 (2012).

41. Perera R, Riley C, Isaac G et al. Dengue virus infection perturbs lipid homeostasis in infected mosquito cells. PLoS Pathog. 8, e1002584 (2012).

42. Rothwell C, Lebreton A, Young Ng C et al. Cholesterol biosynthesis modulation regulates dengue viral replication. Virology 389, 8-19 (2009).

43. Keilian M, Chanel-Vos C, Liao M. Alphavirus entry and membrane fusion. Viruses 2 , 796-825 (2010).

44. Lambrechts L, Scott TW. Mode of transmission and the evolution of arbovirus virulence in mosquito vectors. Proc. Biol. Sci. 276, 1369-1378 (2009).

45. McMeniman CJ, Lane RV, Cass BN et al. Stable introduction of a life-shortening Wolbachia infection into the mosquito Aedes aegypti. Science 323, 141-144 (2009).

46. Yeap HL, Mee P, Walker T et al. Dynamics of the "popcorn" Wolbachia infection in outbred Aedes aegypti informs prospects for mosquito vector control. Genetics 187, 583-595 (2011).

47. McMeniman CJ, O’Neill SL. A virulent Wolbachia infection decreases the viability of the dengue vector Aedes aegypti during periods of embryonic quiescence. PLoS Negl. Trop. Dis. 4, e748 (2010).

48. Moreira LA, Saig E, Turley AP et al. Human probing behavior of Aedes aegypti when infected with a life-shortening strain of Wolbachia. PLoS Negl. Trop. Dis. 3, e568 (2009). 
49. Turley AP, Moreira LA, O'Neill SL, McGraw EA. Wolbachia infection reduces bloodfeeding success in the dengue fever mosquito, Aedes aegypti. PLoS Negl. Trop. Dis. 3, e516 (2009).

50. Hurst TP, Pittman G, O’Neill SL et al. Impacts of Wolbachia infection on predator prey relationships: evaluating survival and horizontal transfer between $w$ MelPop infected Aedes aegypti and its predators. J. Med. Entomol. 49, 624-630 (2012).
51. Turley AP, Zalucki MP, O’Neill SL, McGraw EA. Transinfected Wolbachia have minimal effects on male reproductive success in Aedes aegypti. Parasit. Vectors 6, 36 (2013).

52. Suh E, Mercer DR, Fu Y, Dobson SL. Pathogenicity of life-shortening Wolbachia in Aedes albopictus after transfer from Drosophila melanogaster. Appl. Environ. Microbiol. 75, 7783-7788 (2009).
53. Hancock PA, Sinkins SP, Godfray HC. Strategies for introducing Wolbachia to reduce transmission of mosquito-borne diseases. PLoS Negl. Trop. Dis. 5, e1024 (2011).

54. Zhang G, Hussain M, O'Neill SL, Asgari S. Wolbachia uses a host microRNA to regulate transcripts of a methyltransferase, contributing to dengue virus inhibition in Aedes aegypti. Proc. Natl Acad. Sci. USA 110(25), 10276-10281 (2013). 